\title{
Beobachtungen von kleinen Planeten
}

am Refractor der Sternwarte in Kiel von Prof. E. Lamp.

\begin{tabular}{|c|c|c|c|c|c|c|c|c|c|c|}
\hline Datum & M.Z.Kiel & $\Delta \alpha$ & $\Delta \delta$ & $|\mathrm{Vgl}|$. & $\alpha$ app. & Par. & $\delta$ app. & Par. & Red. ad 1. app. & * \\
\hline 1890 & & & & & Ther & & & & & \\
\hline Dec. 14 & $10^{\mathrm{h}} 56^{\mathrm{m}} 19^{\mathrm{s}}$ & $+5^{\mathrm{m}} 53^{\mathrm{s}} \cdot 60$ & $-8^{\prime} 48.0$ & 4 & $4^{\mathrm{h}} 2 \mathrm{I}^{\mathrm{m}} 43^{\mathrm{s}} \cdot 1 \mathrm{x}$ & +0.01 & $+22^{\circ} 15^{\prime} 3^{\prime \prime \prime} 7$ & $+2 \% 4$ & $+3^{s} \cdot 57$ & I \\
\hline 14 & $10 \quad 5820$ & $\begin{array}{lll}-6 & 27\end{array}$ & +620.6 & 4 & 42143.31 & $+c-1-1$ & +221530.7 & +2.4 & $+3.60+9.9$ & 2 \\
\hline 15 & $10 \quad 50 \quad 48$ & $-7 \times 7.08$ & $+43^{2.6}$ & 4 & 42054 & +0.01 & +221342.9 & +2.4 & $+3.60+10.1$ & 2 \\
\hline 15 & $\begin{array}{lll}1 \mathrm{I} & 6 & \mathrm{I} 3\end{array}$ & +53.85 & -1039.5 & 4 & $42053.3^{6}$ & +0.02 & +221340.4 & +2.4 & $+3.57+11.0$ & \\
\hline
\end{tabular}

I $890-91$

(II) Parthenope.

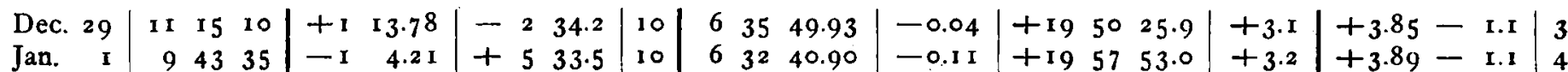

Mittlere Oerter der Vergleichsterne für I 890.0.

\begin{tabular}{|c|c|c|c|}
\hline * & $\alpha 1890.0$ & $\delta 1890.0$ & Autorität \\
\hline $\begin{array}{l}\mathbf{I} \\
2\end{array}$ & 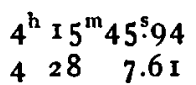 & $\begin{array}{r}+22^{\circ} 24^{\prime} 8^{\prime \prime} 9 \\
+2299.2\end{array}$ & $\begin{array}{l}\text { AG. Berlin } \\
\text { AG. Berlin }\end{array}$ \\
\hline
\end{tabular}

\begin{tabular}{|c|c|c|c|}
\hline 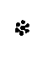 & a 1890.0 & $\delta 1890.0$ & Autorität \\
\hline $\begin{array}{l}3 \\
4\end{array}$ & $\begin{array}{lll}6^{\mathrm{h}} & 34^{\mathrm{m}} & 3^{2 \mathrm{~s}} \cdot 30 \\
6 & 33 & 41.22\end{array}$ & $\begin{array}{l}+19^{\circ} 53^{\prime} \text { 1".2 } \\
+195220.6\end{array}$ & $\begin{array}{l}W_{2} 6^{\mathrm{h}} .982 \\
W_{2} 6.946\end{array}$ \\
\hline
\end{tabular}

Vergleichung der Beobachtungen mit Ephemeriden (B-R).

(24) Themis.

(Berl. Jahrb. I 892.)

\begin{tabular}{c|c|c|}
\hline 1890 & $\Delta \alpha$ & $\Delta \delta$ \\
\hline Dec. 14 & -0.53 & +0.7 \\
14 & -0.26 & -0.1
\end{tabular}

\begin{tabular}{|r|c|c|}
\hline \multicolumn{1}{c|}{1890} & $\Delta \alpha$ & $\Delta \delta$ \\
\hline Dec. 15 & -0.33 & -0.3 \\
15 & -0.56 & -1.6
\end{tabular}

(I I) Parthenope.

(Astr. Nachr. 3008.)

\begin{tabular}{|c|c|c|}
\hline $1890-91$ & $\Delta \alpha$ & $\Delta \delta$ \\
\hline $\begin{array}{lr}\text { Dec. } & 29 \\
\text { Jan } \quad 1\end{array}$ & $\begin{array}{l}+13.30 \\
+0.96\end{array}$ & $\begin{array}{l}-3.1 \\
+1.0\end{array}$ \\
\hline
\end{tabular}

Bemerkungen.

I890 Dec. I 4 und I 5. Beobachtungen an hellen Fäden. - Dec. 29. Beobachtung an Metallfäden ohne künstliche Beleuchtung. - 1891 Jan. I. Beobachtung an den Mikrometerfäden im hellen Feld; diese Beobachtung der Parthenope daher viel sicherer als die vorhergehende.

Die Positionen der Sterne I und 2 sind von Herrn Prof. Becker in Strassburg gütigst mitgetheilt worden.

Kiel 189 r Januar.

E. Lamp.

\section{Mittheilungen über kleine Planeten.}

(243) Ida. Beobachtung von Dr. F. Palisa in Wien : 1891 Febr. $14 \times 10^{\mathrm{h}} 4^{\mathrm{m}} \mathrm{I} 6^{\mathrm{s}} \mathrm{M}$. Z. Wien $\Delta \alpha=+\mathrm{I}^{\mathrm{m}} 5^{\mathrm{s}} 60$ $\Delta \delta=+9^{\prime} 50^{\prime \prime} 4 \alpha$ app. $=10^{\mathrm{h}} 14^{\mathrm{m}} 0^{5} 5^{6}\left(9.266_{\mathrm{n}}\right) . \delta$ app. $=+10^{\circ} 5^{\circ} \mathrm{r} 9^{\prime \prime} 8(0.737)$

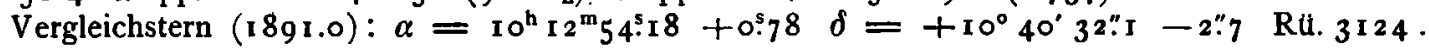

(303) (Charlois Febr. 11), Beobachtung von Dr. W. Luther in Hamburg: $189 \mathrm{r}$ Febr. $131 \mathrm{x}^{\mathrm{h}} 3^{\mathrm{m}} 19^{\mathrm{s}} \mathrm{M}$.Z. Hamb.

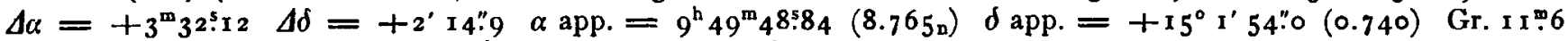

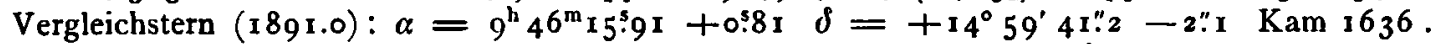

Beobachtung von Dr. F. Palisa in Wien: 1891 Febr. $141_{1}^{\mathrm{h}} 4^{\mathrm{m}} 20^{\mathrm{s}}$ M. Z. Wien $\Delta a=-1^{\mathrm{m}} 44^{\mathrm{s}} .04 \quad \Delta \delta=$ $-8^{\prime} 5^{\prime \prime} .7 \quad \alpha$ app. $=9^{\mathrm{h}} 4^{\mathrm{m}}{ }^{\mathrm{m}} 8^{\mathrm{s}} .33(9.064 \mathrm{n}) \quad \delta$ app. $=+15^{\circ} 5^{\prime} 49^{\prime \prime} . \mathrm{r}(0.687)$

Vergleichstern (1891.0): $\alpha=9^{\mathrm{h}} 5^{0^{\mathrm{m}} 41^{\mathrm{s}} 57+0.80} \delta=+\times 5^{\circ} \times 4^{\prime} 47^{\prime \prime} 9-2$ ". $\quad \mathrm{W}_{2}$ 1037.

(304) (Millosevich Febr. 12). Schreiben von Prof. E. Millosevich in Rom d. d. 13. Februar: Esaminando il cielo per mezzo della Carta No. 4 di Palisa ho ieri sera, dopo aver osservato Hebe, trovato un pianetino di $12^{\mathrm{ma}}$ grandezza, che era all' incirca in opposizione e che potrebbe essere o nuovo o uno dei perduti. Ecco il luogo:

I 891 Febbr. 12 I $2^{\mathrm{h}} 55^{\mathrm{m}} \times 9^{\mathrm{s}}$ t. m. Roma $\Delta \alpha=-0^{\mathrm{m}} 9^{\mathrm{s}} 94^{\prime} \Delta \delta=-5^{\prime} 55^{\prime \prime} 9$ Confr. $7.7 \quad \alpha$ app. $=9^{\mathrm{h}} 5^{\mathrm{r}^{\mathrm{m}} 27^{\mathrm{s}} 20}$ $(8.843) \delta$ app. $=+16^{\circ} 5^{\prime}{ }_{4}^{\prime \prime \prime} 2(0.572)$

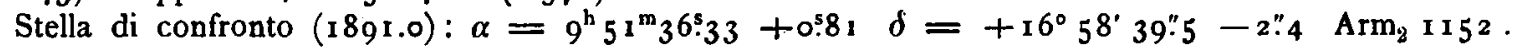

